To appear in Astrophys J. March 10, 2002

\title{
Ambipolar Drift in a Turbulent Medium
}

\author{
Ellen G. Zweibel \\ JILA \& Department of Astrophysical $\&$ Planetary Sciences, University of Colorado, Boulder CO \\ 80309
}

\begin{abstract}
The interstellar magnetic field strength and density are observed to be correlated, but there is a large dispersion in this relation. In particular, the magnetic field is often observed to be weaker than expected. At low ionization fraction, ion-neutral drift, or ambipolar diffusion, permits slip of the field relative to the neutral gas and tends to make the field strength more uniform, but is thought to be too slow to explain the observations. The purpose of this paper is to show that ion-neutral drift is significantly faster in a turbulent medium than in a quiescent one. We suggest that this fast ambipolar diffusion can explain the surprisingly low magnetic fieldstrengths sometimes observed in dense interstellar gas.
\end{abstract}

Subject headings: ISM: magnetic fields, turbulence, MHD

\section{Introduction}

The interstellar magnetic fieldstrength and gas density are observed to be correlated (Troland \& Heiles 1986, Crutcher 1999). This relationship is thought to arise from so-called ideal magnetohydrodynamic (MHD) processes, in which the field is frozen to the ambient medium. Under ideal MHD conditions, if the ratio of mass to magnetic flux were everywhere constant, the slope $q \equiv d \log B / d \log \rho$ of the fieldstrength - density correlation would be unity for compression normal to $\mathbf{B}$, zero for compression parallel to $\mathbf{B}$, and $2 / 3$ for isotropic compression. The observed value of $q$ is approximately 0.5 , which is consistent with equilibrium models of self gravitating clouds which evolved under conditions of frozen flux (Mouschovias 1976, Tomisaka, Ikeuchi, \& Nakamura 1988).

However, a number of observations and upper limits on magnetic fieldstrength suggest that $B \propto \rho^{0.5}$ is more an upper envelope than a scaling law. This is true both in atomic and molecular gas (Bourke et al. 2001, Crutcher 1999, Heiles 2001a, Heiles \& Troland 2001) Although the number of measurements is small, and the field may be underresolved in some cases (Brogan \& Troland 2001), the trend towards weak fields is clear. A different line of argument comes from numerical simulations of molecular clouds: Padoan \& Nordlund (1999) claim that models with weak fields replicate the observations better than models with strong fields. 
Weak fields are difficult to reconcile with ideal MHD. This is particularly so in the case of turbulent molecular clouds. Giant molecular clouds (GMCs) are about 40 times more dense than the mean interstellar gas, suggesting that the magnetic field should be 6-7 times stronger than the mean field. If GMCs are formed by flows parallel to the field then of course the field would not be strengthened, but the mean field is not strong enough to resist compression and collimate the flows. Furthermore, Mestel (1985) has pointed out that accumulation of the mass of a GMC by compression in one dimension requires organized motion of the gas over nearly one kiloparsec, the origin of which is difficult to understand.

Thus, we seek an explanation for the weakness of the magnetic fieldstrength - gas density correlation beyond the scope of ideal MHD. At the very largest lengthscales, ideal MHD should be an excellent approximation. Moving downwards in scale, the first non-ideal effect encountered, at least at low ionization fraction, is ion-neutral drift, or ambipolar diffusion. At the ambipolar scale, which is many orders of magnitude larger than the resistive scale, the magnetic field and plasma become decoupled from the neutral material. This makes it possible to change the mass to flux ratio without altering the magnetic topology. Ambipolar drift has been invoked as the primary magnetic flux transport mechanism in dense, star forming gas since the classic paper by Mestel \& Spitzer (1956). However, it is thought to be too slow to be an effective transport mechanism in diffuse gas (see $\S 2$ for quantitative estimates).

Interstellar gas is turbulent. Turbulent diffusion of quantities such as heat and angular momentum is often invoked in astrophysics as a mechanism for enhancing transport rates above their kinetic theory values, which are usually very slow. Turbulence enhances diffusion rates by mixing the relevant quantity to the small scales at which molecular diffusion operates. This leads to a mixing time which is approximately the eddy turnover time, and is nearly independent of the molecular diffusivity.

Whether turbulence enhances the resistive decay rate of a magnetic field is unclear, because there is substantial evidence that magnetic forces resist stretching the field sufficiently to mix it to the tiny scales at which resistivity operates (Cattaneo \& Vainshtein 1991, Cattaneo 1994, Cattaneo, Hughes, \& Kim 1996). This paper addresses a different question, namely, whether turbulence in a weakly ionized gas can transport magnetic field with respect to the neutral matter, without resistive dissipation necessarily coming into play. Because the ambipolar drift scale is much larger than the resistive scale, the feedback effects which can suppress turbulent resistivity are far less dramatic, although they cannot always be ignored.

We use analytical methods to calculate the mixing rate. Numerical study of mixing requires that numerical diffusion of both field and fluid be very well controlled. Analytical calculations are useful for initial exploration of some of the basic mechanisms.

In $\S 2$, we introduce the physical model, derive an equation for the evolution of the mass to magnetic flux ratio in a weakly ionized medium, estimate relevant timescales, and establish an initial condition. In $\S 3$, we estimate the turbulent diffusion rate based on mixing length theory, 
quantify this estimate with an exact calculation of transport by a wave, and argue that enhanced diffusion requires the introduction of small scales as well as bulk advection. In $\S 4$ we develop a model based on exponential stretching and shrinking. This model leads to a flux redistribution rate which is comparable to the eddy turnover rate, as expected in turbulent diffusion problems. However, the model is two dimensional, and the fluid motions are prescribed without specifically allowing for magnetic forces. In $\S 5$, we consider magnetic feedback. In $\S 6$, we apply the model to the interstellar medium, and discuss the astrophysical constraints imposed by the dynamics. Section 7 is a summary and conclusion. Sections 3 and 4 are relevant to general mixing problems, such as turbulent diffusion of a passive scalar, and the reader who is mainly interested in the astrophysical implications could go directly to $\S 6$.

\section{Basic Equations and Model}

\subsection{Equations for Ambipolar Drift}

We consider a weakly ionized medium with magnetic field $\mathbf{B}$, mass density $\rho$, and ion mass density $\rho_{i} \ll \rho$. We are interested in timescales much longer than the ion-neutral collision time $\tau_{i n}$, in which case the ion-neutral drift $\mathbf{v}_{\mathbf{D}} \equiv \mathbf{v}_{\mathbf{i}}-\mathbf{v}_{\mathbf{n}}$ is well approximated by

$$
\mathbf{v}_{\mathbf{D}}=\frac{(\nabla \times \mathbf{B}) \times \mathbf{B}}{4 \pi \rho_{i}} \tau_{i n}
$$

(Shu 1983).

The magnetic field evolves according to the magnetic induction equation for a perfectly conducting medium

$$
\frac{\partial \mathbf{B}}{\partial t}=\nabla \times\left(\mathbf{v}_{\mathbf{i}} \times \mathbf{B}\right),
$$

Replacing $\mathbf{v}_{\mathbf{i}}$ with $\mathbf{v}_{\mathbf{D}}+\mathbf{v}_{\mathbf{n}}$, and approximating $\mathbf{v}_{\mathbf{n}}$ by the center of mass velocity $\mathbf{v}$, we rewrite the induction equation as

$$
\frac{\partial \mathbf{B}}{\partial t}=\nabla \times(\mathbf{v} \times \mathbf{B})+\nabla \times\left(\mathbf{v}_{\mathbf{D}} \times \mathbf{B}\right),
$$

where $\mathbf{v}_{\mathbf{D}}$ is given by eqn. (1). The first term on the RHS of eqn. (3) can be expanded using the identity $\nabla \times(\mathbf{v} \times \mathbf{B})=\mathbf{B} \cdot \nabla \mathbf{v}-\mathbf{v} \cdot \nabla \mathbf{B}-\mathbf{B} \nabla \cdot \mathbf{v}$. Then, using the continuity equation

$$
\frac{\partial \rho}{\partial t}=-\mathbf{v} \cdot \nabla \rho-\rho \nabla \cdot \mathbf{v}
$$

we derive an evolution equation for the magnetic field to density ratio $\mathbf{B} / \rho$

$$
\frac{\partial}{\partial t} \frac{\mathbf{B}}{\rho}+\mathbf{v} \cdot \nabla \frac{\mathbf{B}}{\rho}=\frac{\mathbf{B}}{\rho} \cdot \nabla \mathbf{v}+\frac{1}{\rho} \nabla \times\left(\mathbf{v}_{\mathbf{D}} \times \mathbf{B}\right) .
$$

The left hand side of eqn. (5) is the comoving, or convective, time derivative of $\mathbf{B} / \rho$. The first term on the right hand side represents stretching of the fieldlines, and is a consequence of the frozen 
flux condition. The second term on the right hand side represents the evolution of $\mathbf{B} / \rho$ caused by ambipolar drift.

We now restrict ourselves to two dimensional, incompressible flows perpendicular to a straight magnetic field. This geometry captures the main effects under study, and is consistent with the nature of turbulence in a strong, well ordered magnetic field (Strauss 1976, Sridhar \& Goldreich 1994, Goldreich \& Sridhar 1997). We recognize that interstellar turbulence is frequently observed to be highly supersonic, and thus cannot be entirely incompressible. We expect that compressible turbulence to result in magnetic flux transport just as incompressible turbulence does, but that magnetic feedback on the turbulence is stronger in the compressible case.

Under the assumptions of incompressibility and two dimensionality, the line stretching term vanishes, and eqn. (5) reduces to

$$
\frac{\partial}{\partial t} \frac{B}{\rho}+\mathbf{v} \cdot \nabla \frac{B}{\rho}=\frac{1}{\rho} \nabla \cdot \frac{B^{2}}{4 \pi \rho} \tau_{n i} \nabla B
$$

where $B$ now represents the amplitude of the magnetic field, $\tau_{n i} \equiv \tau_{i n} \rho_{n} / \rho_{i}$ is the neutral-ion collision time, and we have used eqn. (1). Equation (6) is close to an advection - diffusion equation for the flux to mass ratio $Q \equiv B / \rho$

$$
\frac{\partial Q}{\partial t}+\mathbf{v} \cdot \nabla Q=\frac{1}{\rho} \nabla \cdot \lambda \nabla B
$$

provided that we define the diffusivity $\lambda$ as $\tau_{n i} v_{A}^{2}$, where $B /(4 \pi \rho)^{1 / 2}$ is the Alfven speed.

The diffusion of $Q$ is nonlinear in the sense that $\lambda=\lambda(Q, \rho)$. This nonlinearity can produce sharp fronts along surfaces where $B$ vanishes or is tightly folded (Brandenburg \& Zweibel 1994), similar to fronts created by nonlinear thermal conduction (Zel'dovich \& Raizer 1966). Resistive diffusion in these current sheets alters the mass to flux ratio as well as changing the magnetic topology (Brandenburg \& Zweibel 1995, Zweibel \& Brandenburg 1997). In this paper we assume that the relative variation of $B / \rho$ is so weak that nonlinear effects play only a minor role in ambipolar drift, and $B$ remains nonsingular.

Equation (7) can be used to derive an equation for the rate of change of $B$ within a comoving volume $V$ of fluid; that is, a fluid element of fixed mass. We have

$$
\frac{d}{d t} \int_{V} d^{3} x \rho Q=\int_{V} d^{3} x \frac{\partial}{\partial t} \rho Q+\int_{S} d^{2} x \rho Q \mathbf{v} \cdot \hat{\mathbf{n}},
$$

where $S$ is the surface of $V$. The first term on the RHS of eqn. (8) is the Eulerian change of $B$ and the second term accounts for the motion of $V$. Using eqns. (4) and (7) to expand the first term on the RHS and applying Gauss' theorem yields

$$
\frac{d}{d t} \int_{V} d^{3} x \rho Q=\int_{S} d^{2} x \lambda \hat{\mathbf{n}} \cdot \nabla B
$$

Since the mass within $V$ is constant, eqn. (9) shows that the flux to mass ratio within a volume moving with the center of mass velocity decreases if the magnetic field decreases outward on the surface of the volume. 


\subsection{Timescales}

In the absence of flow, the characteristic diffusion time for a magnetic field of representative strength $B$ and scale length $L \sim|B / \nabla B|$ is

$$
t_{d 0} \equiv \frac{L^{2}}{\lambda}=\frac{L^{2}}{v_{A}^{2} \tau_{n i}}=\frac{\tau_{A}^{2}}{\tau_{n i}}, .
$$

where $\tau_{A} \equiv L / v_{A}$ is the global Alfven time.

Expressing $t_{d 0}$ in physical units reveals the magnitude of the timescale problem. We take $\tau_{n i}$ from Draine, Dalgarno, \& Roberge (1983); when the ratio of ion to neutral atomic weight $A_{i} / A_{n} \gg 1, \tau_{n i}=6.7 \times 10^{8} n_{i}^{-1} \mathrm{~s}$. The Alfven speed $v_{A}=2.2 \times 10^{5} B_{\mu} /\left(n_{n} A_{n}\right)^{1 / 2} \mathrm{~cm} \mathrm{~s}^{-1}$. The diffusivity $\lambda$ is then $3.2 \times 10^{19} B_{\mu}^{2} /\left(A_{n} n_{n}^{2} x_{i}\right) \mathrm{cm}^{2} \mathrm{~s}^{-1}$, where $x_{i} \equiv n_{i} / n_{n}$ is the ionization fraction. The drift time is

$$
t_{d 0}=3.1 \times 10^{20} \frac{N_{20}^{2} x_{i} A_{n}}{B_{\mu}^{2}} \mathrm{~s},
$$

where $N_{20} \equiv n_{n} L$ is the column density in units of $10^{20} \mathrm{~cm}^{-2}$. For example, the systems reported by Heiles (2001) have $N_{20}$ a few tenths to a few, with $B_{\mu}$ typically 3 . If we take $A_{n}=1.4$ for gas of cosmic composition and $x_{i}=10^{-4}$, which is probably a conservative lower limit, then we find $t_{d 0}$ is of order $10^{8} \mathrm{yr}$ for these systems. Since this is much more than the $10^{6}-10^{7} \mathrm{yr}$ expected lifetime of an interstellar cloud, the diffusion rate must be enhanced by a factor of 10 - 100 in order to explain the flatness of the $B-n$ relation.

Mixing by eddies in the neutral gas requires that the magnetic field be frozen to the flow. The degree of freezing is measured by the ambipolar Reynolds number $R_{A D}$, which is large under frozen in conditions (Zweibel \& Brandenburg 1997). For eddies of characteristic size $l$ and speed $v_{t}$,

$$
R_{A D} \equiv \frac{l v_{t}}{\lambda}
$$

Thus, the field is frozen to the turbulent flow for

$$
\left(\frac{l}{L}\right)\left(\frac{v_{t}}{v_{A}}\right)>\frac{\tau_{n i}}{\tau_{A}}
$$

Since $\tau_{n i} / \tau_{A}$ is expected to be small, while $v_{t} / v_{A}$ is order unity, eqn. (13) implies a fair degree of dynamic range for the sizes of eddies that can mix the field. Equation (12) can also be written as

$$
R_{A D}=\frac{v_{t}^{2}}{v_{A}^{2}} \frac{\tau_{d}}{\tau_{n i}},
$$

which shows that if the flow is at or above equipartition with the field $\left(v_{t} \geq v_{A}\right)$, the field is frozen in if the neutral-ion collision time is less than the eddy turnover time. 


\subsection{Initial Condition}

It will be useful in the following analysis to have a definite model for $B$. We take as an initial condition

$$
B(x, y, 0)=B_{00}+\frac{1}{2} B_{0}^{\prime \prime} x^{2},
$$

where $B_{00}$ and $B_{0}^{\prime \prime}<0$ are constants. We will define $\lambda$ using $B_{00}$ for $B_{0}$ in quantitative examples. We view eqn. (15) as the first two terms in a Taylor expansion of a magnetic field which peaks at $x=0$, and will assume $x / L \ll 1$.

We first consider pure diffusion. Motivated by the initial condition (15), we seek solutions of eqn. (7), with $\mathbf{v}=0$, of the form

$$
B(x, y, t)=B_{0}(t)+\frac{1}{2} B_{0}^{\prime \prime} x^{2} .
$$

Substituting eqn. (16) into eqn. (7) and using eqn. (15), we find

$$
B_{0}(t)=B_{00}+B_{0}^{\prime \prime} \lambda t
$$

Equation (17) predicts that the peak field decreases by a factor of two on a timescale

$$
t_{d 0} \equiv-B_{00} /\left(2 \lambda B_{0}^{\prime \prime}\right)
$$

Equation (18) agrees with eqn. (10) if we define the magnetic lengthscale $L$ by

$$
L=\left(-\frac{B_{00}}{2 B_{0}^{\prime \prime}}\right)^{1 / 2} .
$$

\section{Diffusion in the Presence of Waves}

We begin with a mixing length argument. Consider a magnetic flux tube of width $a$ which is carried by a random flow $u$ a distance $l$ down the gradient of $B$. The field in the tube diffuses into the ambient medium; thus the motion causes net transport of $B$. The transport is most effective when the diffusion time across the tube is comparable to the advection time

$$
\frac{a^{2}}{\lambda} \sim \frac{l}{u}
$$

because if $a^{2} / \lambda \gg l / u$, the flux tubes return to their original positions with nearly the same value of the field, while if $a^{2} / \lambda \ll l / u$ the field diffuses too quickly to be advected by the flow.

Advection spreads $B$ over a distance $l$ in a time $l / u$. In this time, $B$ would spread diffusively

over a distance $(\lambda l / u)^{1 / 2}$. By eqn. (20), this distance is just $a$. Advective mixing accelerates the transport of $B$ only if $a / l<1$, meaning that the motion consists of thin fingers which travel much 
further than their widths (see Ottino 1989 for discussion of mixing by fingers, or tendrils). Such fingers are not seen in models of Alfvenic turbulence, and it is not clear that they would form in a weakly stratified gas such as the interstellar medium. Interpreted more broadly, the argument presented here shows that turbulent mixing requires more than just advection and dispersal; it also requires the formation of small scales.

Now, we quantify this result. Weak turbulence theory, in which turbulence is modelled as a superposition of randomly phased waves, can be used to compute the rate of turbulent diffusion (eg. Moffatt 1978, Gruzinov \& Diamond 1994). In this so-called quasilinear approach, one partitions quantities into mean and fluctuating parts and calculates the average effect of the fluctuations on the mean part. We used this method in a previous study of ambipolar diffusion (Zweibel 1988). In the present problem it is possible, as well as instructive, to solve the induction equation exactly instead of averaging it. This confirms the argument given in $\S 2$.

We introduce a periodic flow in the $\hat{x}$ direction

$$
\mathbf{v}=\hat{x} u \sin \omega t \sin k y .
$$

Motivated by the initial condition eqn. (15), we try a solution of the advection-diffusion equation (7) of the form

$$
B(x, y, t)=B_{0}(t)+\frac{1}{2} B_{0}^{\prime \prime}+B_{1}(t) x \sin k y+B_{2}(t) \cos 2 k y .
$$

Substituting eqn. (22) into eqn. (7), using eqn. (21), and equating like powers of $x$ and Fourier harmonics of $y$ leads to a set of coupled ODEs for the functions $B_{0}, B_{1}$, and $B_{2}$. The solution for $B_{0}$, which follows the decay of the peak magnetic field, is

$$
\begin{aligned}
B_{0}= & B_{00}+\lambda B_{0}^{\prime \prime} t \\
& +\frac{B_{0}^{\prime \prime} u^{2}}{2\left(\omega^{2}+\Gamma^{2}\right)}\left[\frac{\Gamma}{2}\left(t-\frac{\sin 2 \omega t}{2 \omega}\right)+\frac{\sin ^{2} \omega t}{2}\right. \\
& +\frac{\omega^{2}}{\omega^{2}+\Gamma^{2}}\left(1-e^{-\Gamma t} \cos \omega t\right) \\
& \left.-\frac{\omega \Gamma}{\omega^{2}+\Gamma^{2}} e^{-\Gamma t} \sin \omega t\right]
\end{aligned}
$$

where $\Gamma \equiv \lambda k^{2}$.

The maximum decay rate occurs when the motion given by eqn. (21) is coherent over many wave periods. The long time behavior of $B_{0}$ is then given by

$$
B_{0}=B_{00}+\left(\lambda+\lambda_{t}\right) B_{0}^{\prime \prime} t
$$

where

$$
\lambda_{t} \equiv \frac{\Gamma u^{2}}{4\left(\omega^{2}+\Gamma^{2}\right)}
$$


represents diffusion brought about by advective transport. Equation (25) closely resembles the turbulent diffusivity calculated from quasilinear theory (Moffatt 1978). Maximizing $\lambda_{t}$ with respect to $k$, we find that the maximum occurs for $\omega=\Gamma$, as we asserted in the mixing length argument following eqn. (10), and is

$$
\lambda_{t, \max }=\frac{u^{2}}{8 \lambda k^{2}},
$$

where we have replaced $\Gamma$ by $\lambda k^{2}$. If we express $u$ in terms of the maximum fluid displacement $a \equiv u / \omega$ and take the ratio of $\lambda_{t, \max }$ to $\lambda$, the result is

$$
\frac{\lambda_{t, \max }}{\lambda}=\frac{k^{2} a^{2}}{8}
$$

Equation (27) shows that the diffusion rate is appreciably enhanced by waves only if $k a \gg 1$, meaning that the flow consists of long, thin streamers (see also Press \& Rybicki 1981). We reached the same conclusion from mixing length theory. The missing ingredient is stretching and shrinking of scales, an intrinsic feature of turbulent flows which we incorporate in the next section.

\section{Stagnation Point Flow}

Hyperbolic stagnation point flow is a particularly tractable example of a flow with exponential shrinking and stretching. At hyperbolic stagnation points, the fluid flow converges in one (or two) directions and diverges in the other direction(s), while maintaining incompressibility. It is

well known that diffusion is accelerated in the vicinity of stagnation points, due to the shrinking of scales in the convergent directions (Moffatt 1978, Zweibel 1998), while Zel'dovich et al. (1984) demonstrated dynamo action by a random ensemble of stagnation points. The role of hyperbolic stagnation points in the mixing of scalar fields in turbulent flows has recently been reviewed (Shraiman \& Siggia 2000) with emphasis on the development of intermittency, and the high order moments of the distribution of concentrations.

The advection-diffusion problem for fields of the form (15) is exactly soluble for stagnation point flow. In subsection (4.1) we compute the effect of a single stagnation point. In subsection (4.2) we superimpose the effects of a random ensemble of stagnation points, and in subsection (4.3) we discuss the relationship between the stagnation point model and turbulent flow. Our model is not intended to be a full theory of turbulence, but merely illustrative.

\subsection{A Single Stagnation Point}

We consider two dimensional, incompressible flow near a stagnation point at $\left(a_{x}, a_{y}\right)$. For fields of the form given by eqn. (15), we require $a_{x} / L \ll 1$, where $L$ is given by eqn. (19). The flow is

$$
\begin{gathered}
v_{x}=-\gamma\left(x-a_{x}\right), \\
v_{y}=\gamma\left(y-a_{y}\right),
\end{gathered}
$$


where $\gamma$ is a constant. It is straightforward to integrate equations (28) to find the position at time $t$ of a fluid parcel which is at position $\left(x_{0}, y_{0}\right)$ at $t=0$

$$
\begin{gathered}
x=a_{x}+\left(x_{0}-a_{x}\right) e^{-\gamma t} \\
y=a_{y}+\left(y_{0}-a_{y}\right) e^{\gamma t} .
\end{gathered}
$$

The initial coordinates in terms of the coordinates at time $t$ are

$$
\begin{gathered}
x_{0}=a_{x}+\left(x-a_{x}\right) e^{\gamma t} \\
y_{0}=a_{y}+\left(y-a_{y}\right) e^{-\gamma t} .
\end{gathered}
$$

We now compute the effect of this stagnation point flow on the diffusion of the magnetic field. With eqn. (15) as the initial condition, we look for a solution of the form

$$
B(x, y, t)=B_{0}(t)+\frac{1}{2} B_{0}^{\prime \prime} x_{0}^{2}
$$

where $x_{0}(x, y, t)$ is given by eqn. (32). Substituting eqn. (34) into the advection-diffusion equation (7) yields

$$
\dot{B}_{0}=\lambda B_{0}^{\prime \prime} e^{2 \gamma t}
$$

$B$ is of the form (34) exist for two reasons. First, $x_{0}$ is a constant of the motion, so for any function $f\left(x_{0}\right)$

$$
\frac{\partial f\left(x_{0}\right)}{\partial t}+\mathbf{v} \cdot \nabla f\left(x_{0}\right)=0
$$

where $\mathbf{v}$ is given by eqns. (28). Second, eqn. (32) shows that $x_{0}$ is a linear function of $x$ and $y$, so $\nabla^{2} x_{0}$ is only a function of time, and is independent of the stagnation point location $\left(a_{x}, a_{y}\right)$.

The solution of eqn. (35) which fits the initial conditions is

$$
B_{0}(t)=B_{00}+\frac{\lambda}{2 \gamma} B_{0}^{\prime \prime}\left(e^{2 \gamma t}-1\right) .
$$

The location of the peak field evolves in time to $a_{x}\left(1-e^{-\gamma t}\right)$, but this is irrelevant because the density $\rho$ is shifted by the same amount. It is only diffusion which affects the mass to flux ratio.

According to eqn. (35), the peak field has dropped to half its value in the time $t_{\gamma}$

$$
t_{\gamma}=\frac{1}{2 \gamma} \ln \left(1+2 \gamma t_{d 0}\right)
$$

where $t_{d 0}$ is the diffusion time in the static case, defined in eqn. (18). Equation (38) shows that the diffusion time depends only logarithmically on the diffusivity $\lambda$. This arises because of the exponential growth of the magnetic field gradient, as seen in eqn. (35).

The quantity $2 \gamma t_{d 0}$ which appears in the logarithm in eqn. (38) is, however, a large number. If we identify $\gamma^{-1} \sim l / v_{t}$ with an eddy turnover time $\tau_{d}$, and use eqns. (10) and (12), we can write

$$
2 \gamma t_{d 0} \sim 2 \frac{\tau_{A}^{2}}{\tau_{d} \tau_{n i}} \sim 2 R_{A D} \frac{L^{2}}{l^{2}}
$$


At the time $t_{\gamma}$, the $\hat{x}$ component of the drift velocity $v_{D x}$ is half the flow velocity $v_{x}=\gamma x$. Beyond this time, the magnetic field is not well coupled to the flow.

\subsection{An Ensemble of Stagnation Points}

We compute the evolution of the magnetic field carried by Lagrangian fluid elements under sequences of stagnation point flows oriented in random directions, each one of which endures for a time $\tau$ (see Childress \& Gilbert 1995 for a general discussion of these so-called renewing flows).

We take the flow during time $(n-1) \tau<t<n \tau$ to be

$$
\begin{aligned}
& v_{n x}=\gamma \mu_{n} x+\gamma\left(1-\mu_{n}^{2}\right)^{1 / 2} y, \\
& v_{n y}=\gamma\left(1-\mu_{n}^{2}\right)^{1 / 2} x-\gamma \mu_{n} y,
\end{aligned}
$$

where $-1 \leq \mu_{n} \leq 1$ and $n \geq 1$, and for simplicity we have taken all flows to have the same strength $\gamma$. Equation (40) reduces to eqn. (28) if $\mu=-1$. These flows are curl free, and hence not of the most general possible type. However, in the neighborhoods of stagnation points, vorticity leads to changes of scale at an algebraic rather than exponential rate, complicating the mathematics while having little effect on the diffusion rate (Zel'dovich et al. 1984, Zweibel 1998). Thus, vorticity is of secondary importance to our problem, and we omit it here, although interstellar turbulence almost certainly possesses vorticity.

Let the $(x, y)$ coordinates of a fluid parcel at time $(n-1) \tau$ be $\mathbf{r}_{\mathbf{n}-\mathbf{1}}$. Then at time $n \tau$, the coordinates $\mathbf{r}_{\mathbf{n}}$ can be written as

$$
\mathbf{r}_{\mathbf{n}}=\mathbf{A}_{\mathbf{n}} \cdot \mathbf{r}_{\mathbf{n}-\mathbf{1}}
$$

where the matrix $\mathbf{A}_{\mathbf{n}}$ is

$$
\left(\begin{array}{cc}
\cosh \gamma \tau+\mu_{n} \sinh \gamma \tau & \left(1-\mu_{n}^{2}\right)^{1 / 2} \sinh \gamma \tau \\
\left(1-\mu_{n}^{2}\right)^{1 / 2} \sinh \gamma \tau & \cosh \gamma \tau-\mu_{n} \sinh \gamma \tau
\end{array}\right)
$$

Inverting eqn. (42) yields $\mathbf{r}_{\mathbf{n}-\mathbf{1}}$ in terms of $\mathbf{r}_{\mathbf{n}}$

$$
\mathbf{r}_{\mathbf{n}-1}=\mathbf{A}_{\mathbf{n}}^{-1} \cdot \mathbf{r}_{\mathbf{n}}
$$

Successive backwards iteration of eqn. (43) yields the initial position $\mathbf{r}_{\mathbf{0}}$ in terms of the coordinate $\mathbf{r}_{\mathbf{N}} \equiv \mathbf{r}\left(N \tau, \mathbf{r}_{\mathbf{0}}\right)$

$$
\mathbf{r}_{\mathbf{0}}=\mathbf{A}_{\mathbf{1}}{ }^{-1} \cdot \mathbf{A}_{\mathbf{2}}{ }^{-1} \ldots \mathbf{A}_{\mathbf{N}}{ }^{-1} \cdot \mathbf{r}_{\mathbf{N}}
$$

At times $n \tau<t<(n+1) \tau$, the position $\mathbf{r}(t)$ is related to the postion at time $n \tau$ by an equation similar to eqn. (42), where in the matrix $\mathbf{A}$ we replace $\gamma \tau$ by $\gamma(t-n \tau)$.

Since the matrices $\mathbf{A}_{\mathbf{n}}$ are independent of the spatial coordinates, $\mathbf{r}_{\mathbf{0}}$ is a linear function of $\mathbf{r}_{\mathbf{N}}$, or, more generally, $\mathbf{r}(t)$. This means that $\nabla^{2} x_{0}^{2}$ is a function only of time. It follows that the 
solution $B$ of the advection- diffusion equation (7) can still be written in the form of eqn. (34), and that the rate of diffusion increases with time at the same rate as $\nabla^{2} x_{0}^{2}$.

In order to estimate the rate of increase of $\nabla^{2} x_{0}^{2}$, we generated random sequences of $\mu_{n}$ and calculated $\mathbf{r}_{\mathbf{0}}$ from $\mathbf{r}_{\mathbf{N}}$ according to eqn. (44). We used the result to calculate $\nabla^{2} x_{0}^{2}$ as a function of $n$, or equivalently, as a function of time, since $n$ corresponds to the time $n \tau$. The only adjustable parameter in these calculations is $\gamma \tau$, which measures the coherence of the flow, here the renewal interval in units of the stretching rate. We expect $\gamma \tau$ to be $\mathcal{O}(1)$.

Figure (1) shows the dimensionless diffusion rate as a function of iteration number in the case $\gamma \tau=0.5$. On average, the increase in diffusion rate is well fit by an exponential, and is about $84 \%$

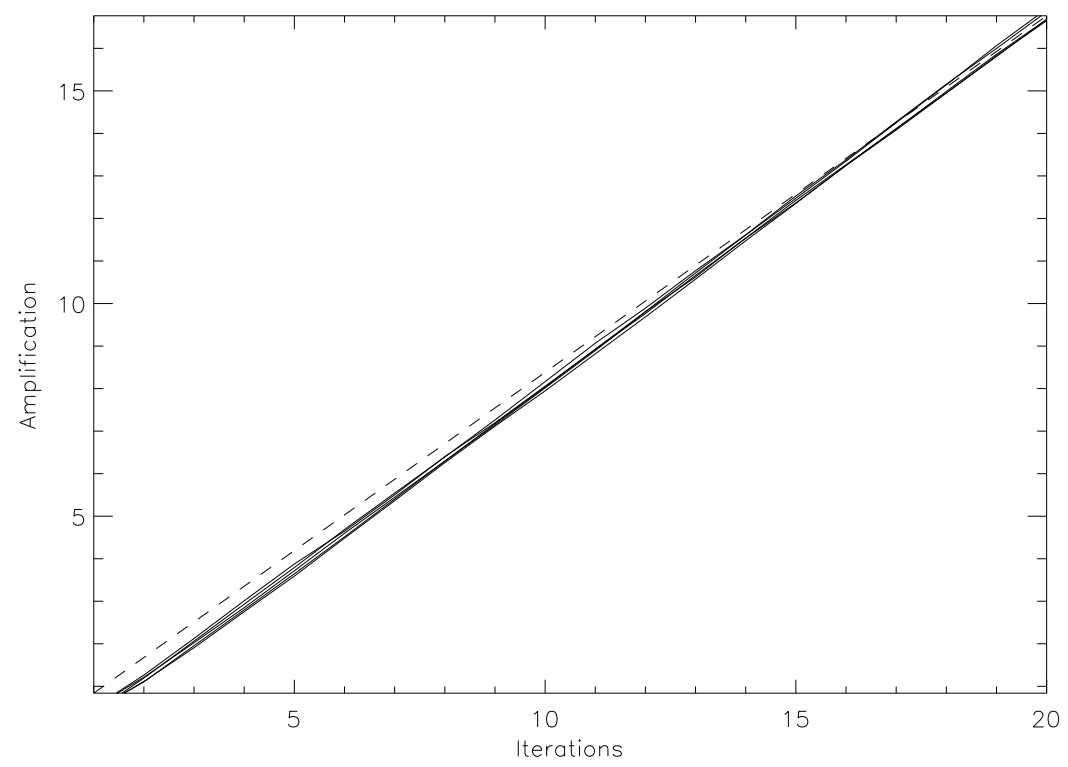

Fig. 1. - The natural log of the increase in diffusion rate, or amplification factor, with time, or the number of iterations. Each solid curve is the average of 50 independent realizations of the iteration process. The dashed line, which has slope 0.838 , is the exponential with the same final value as the average of the curves. The maximum amplification factor possible would occur if all stagnation points had inflow along the $\hat{x}$ axis, and would have a slope of 1 in these units.

the rate of increase for coherent stagnation point flow given in eqn. (28). After 20 renewals the average diffusion rate is more than $10^{7}$ times larger than its original value. This is much larger than the 2-3 orders of magnitude that we estimated in $\S 2.2$ as required to explain the $B-n$ relation. The mean amplification rate is relatively insensitive to the coherence parameter $\gamma \tau$, being about $75 \%$ of maximum if $\gamma \tau=0.1$ and about $87 \%$ of maximum if $\gamma \tau=1$.

However, there is substantial dispersion about the mean. Each solid curve represents 50 
independent sequences of iterations, and Fig. (1) shows differences between them. The standard deviation within each set of 50 sequences is typically about $40 \%$ of the mean, and the amplification factors for single sequences rarely grow exponentially. This is illustrated in Figure (2). The large

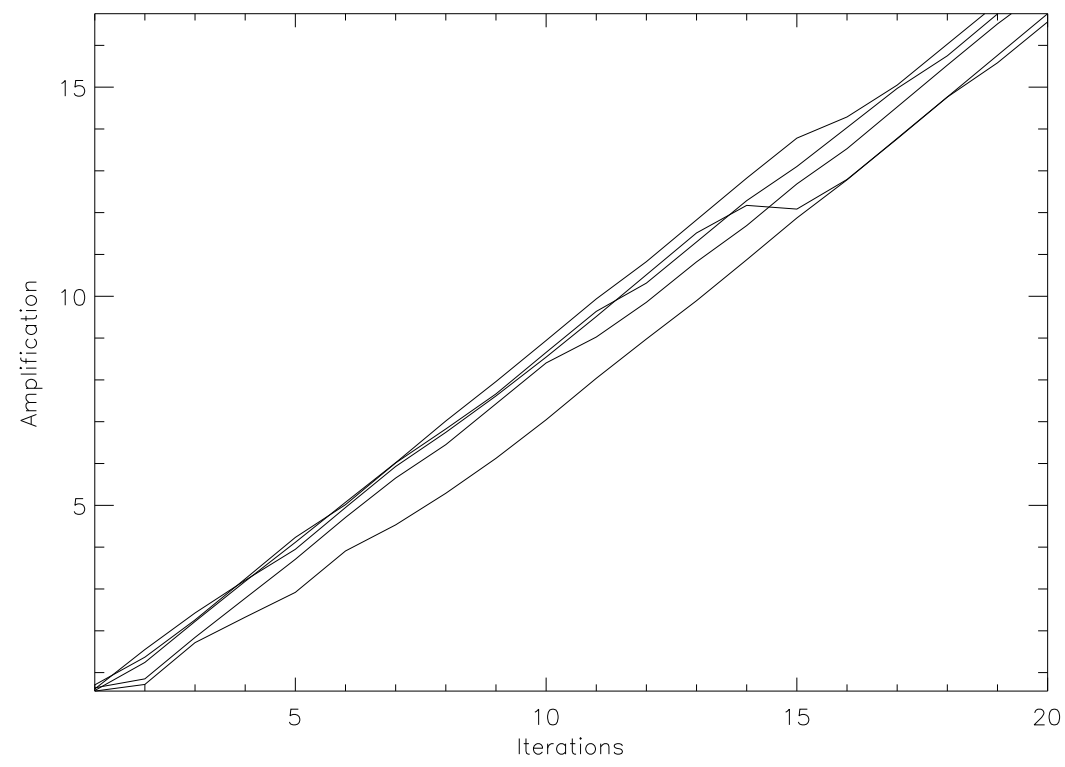

Fig. 2. - The natural log of the increase in diffusion rate, or amplification factor, with time, or the number of iterations. Each solid curve is a single independent realization of the iteration process. The curves, which correspond to the first 5 members of a larger ensemble, show the intrinsic variability of the amplification process.

standard deviation suggests that the diffusion rate in this model is highly intermittent, which is characteristic of turbulent mixing. Additional evidence of intermittency is seen in the PDFs of the distribution of amplification factors, shown in Figure (3) for two different values of $\gamma \tau$. The maximum possible amplification rate of 20 imposes a cutoff on the high amplification side of the curve for $\gamma \tau=1.0$; the PDF for the case $\gamma \tau=0.1$ is more symmetrical because the mean amplification rate is well below the maximum.

\subsection{The Stagnation Point Model and Turbulent Flow}

The hyperbolic stagnation point model achieves fast diffusion by increasing the magnetic field gradient at, on average, nearly exponential rates. Chaotic flow achieves fast stretching and shrinking without hyperbolic stagnation points because the trajectories of neighboring fluid particles separate at an exponential rate. Small differences in the rates of exponential change lead to highly intermittent distributions of scalar quantities, which we saw reflected in the stagnation point model through 


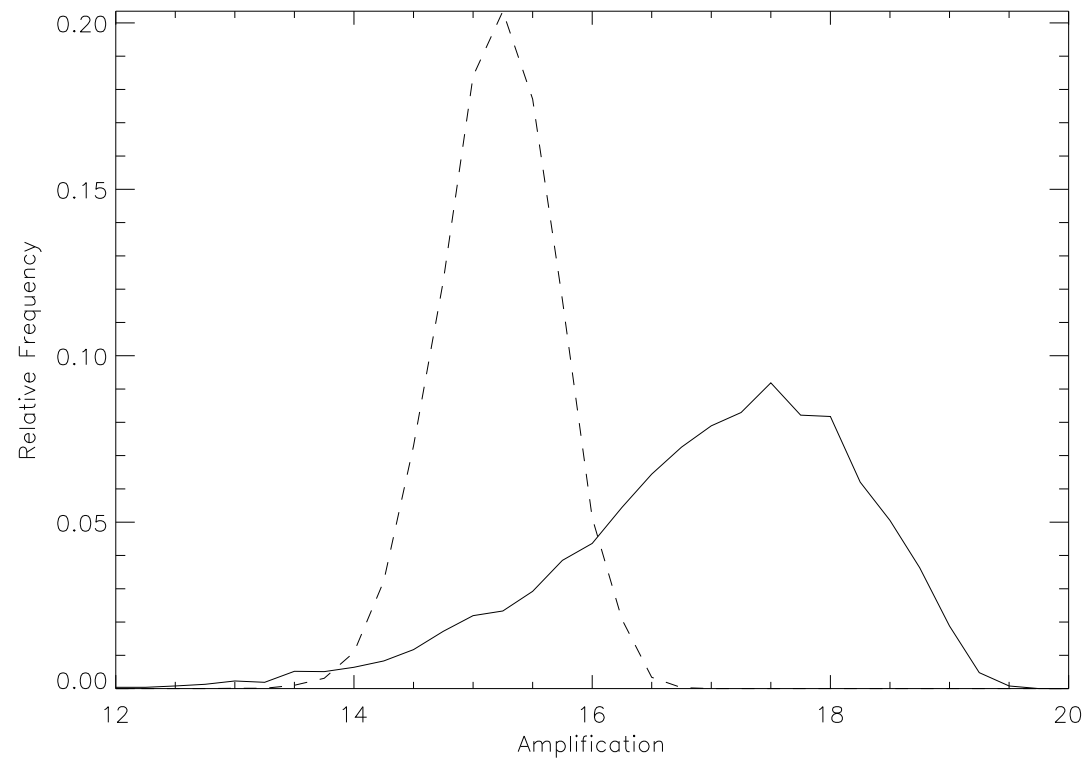

Fig. 3.- Normalized frequency distributions, or PDFs, of the natural logarithms of the amplification factors for 10 iterations at $\gamma \tau=1.0$ (solid curve) and 100 iterations at $\gamma \tau=0.1$ (dashed curve), so that the curves correspond to the same total time. Each curve is based on $10^{4}$ random sequences of iterations.

the wide dispersion of amplification rates (see Figures $2 \& 3$ ). The maximum rate of stretching of a fluid element labelled by its initial position $\mathbf{x}_{\mathbf{0}}$ is given by the Lyapunov exponent $\Lambda\left(\mathbf{x}_{\mathbf{0}}\right)$

$$
\Lambda\left(\mathbf{x}_{\mathbf{0}}\right) \equiv \max _{\mathbf{e}_{\mathbf{0}}} \limsup _{t \rightarrow \infty} \frac{1}{t} \ln \left|\frac{\partial \mathbf{x}}{\partial \mathbf{x}_{\mathbf{0}}} \cdot \mathbf{e}_{\mathbf{0}}\right|
$$

where the $\mathbf{e}_{\mathbf{0}}$ are the set of all possible unit vectors (see Childress \& Gilbert 1995).

In order to make it plausible that the diffusion rate is enhanced by exponential shrinking and stretching in a flow, we imagine that the diffusivity $\lambda$ is so small that we can ignore it. In this limit, the solution of the advection equation for the initial condition (15) is

$$
B(\mathbf{x}, t)=B\left(\mathbf{x}_{\mathbf{0}}, 0\right)=B_{00}+\frac{1}{2} B_{0}^{\prime \prime} x_{0}^{2}(\mathbf{x}) .
$$

If we restore diffusion, it appears in the advection-diffusion equation (7) as the term

$$
\lambda B_{0}^{\prime \prime} \nabla^{2} \frac{x_{0}^{2}}{2}=\lambda B_{0}^{\prime \prime}\left(x_{0} \nabla^{2} x_{0}+\nabla x_{0} \cdot \nabla x_{0}\right) .
$$

The quantity $\nabla x_{0} \cdot \nabla x_{0}$ on the right hand side of eqn. (47) is the square of the inverse of the stretching rate. This suggests heuristically that the diffusion rate grows exponentially. 
Examples of 2D, chaotic, spatially periodic flow with a single lengthscale, including maps of Lyapunov exponents and other measures of chaos, are given by Galloway \& Proctor (1992), Ponty et al. (1993) and Cattaneo et al. (1995).

\section{Dynamical Feedback with Extension to 3D}

In $\S 4$, we prescribed a strictly $2 \mathrm{D}$ flow, and neglected feedback by Lorentz forces. In fact, by virtue of eqn. (1), the diffusion rate cannot be enhanced without increasing the Lorentz force, while 3D effects modify the diffusion process itself (compare eqns. (3) and (6)). In this section we quantify the effects of magnetic feedback and derive criteria for the validity of the $2 \mathrm{D}$ model.

We assume the stagnation point flow given by eqns. (28) with $\mathbf{a}=0$. The magnetic field can be written using eqns. (32), (34), and (37) as

$$
B=B_{00}\left[1-\frac{1}{4 \gamma t_{d 0}}\left(e^{2 \gamma t}-1\right)-\frac{x^{2}}{4 L^{2}} e^{2 \gamma t}\right] .
$$

We evaluate the importance of feedback by following the force on a fluid element over the mixing time $t_{\gamma}$. This probably overestimates the effects of magnetic feedback, because the coherence time $\tau$ of any particular realization of the flow is expected to be less than $t_{\gamma}$. Thus, the constraints on the turbulence which we derive are likely to be conservative. In fact, mixing appears to take place in fully self consistent models of Alfven wave turbulence; Maron \& Goldreich (2001).

In what follows, it is useful to remember that the parameter $2 \gamma t_{d 0}$, the ratio of the classical ambipolar diffusion time to the eddy turnover time, is large; see eqn. (39). We will sometimes use the inverse of this quantity as an expansion parameter.

\subsection{Magnetic Pressure Forces}

We estimate the deceleration of an element of fluid by magnetic pressure in time $t_{\gamma}$. The $\hat{x}$ component of magnetic pressure force $F_{m}$ is

$$
F_{m}=-\hat{x} \frac{\partial}{\partial x} \frac{B^{2}}{8 \pi}
$$

The deceleration $\Delta v^{P}$ of a fluid element over a time $t$ is

$$
\Delta v^{P}\left(x_{0}\right)=\frac{1}{\rho} \int_{0}^{t} d s F_{m}(x(s), s),
$$

where $x(s)=x_{0} e^{-\gamma s}$ is the position of the fluid element at time $s$ and $x_{0}$ is its original position. Substituting eqn. (48) into eqn. (49) and integrating eqn. (50) to $t=t_{\gamma}$ yields to leading order in $2 \gamma t_{d 0}$

$$
\Delta v^{P}\left(x_{0}\right)=-\frac{B_{00}^{2} x_{0}}{16 \pi \rho L^{2} \gamma}\left(2 \gamma t_{d 0}\right)^{1 / 2}
$$


where we have assumed $x_{0} / L \ll 1$.

The average velocity $\bar{v}\left(x_{0}\right)$ of the fluid element over this time is

$$
\bar{v}\left(x_{0}\right) \equiv \frac{1}{t_{\gamma}} \int_{0}^{t_{\gamma}} d s v(x(s), s)
$$

where $x(s)$ is once again the Lagrangian position of a fluid element. For the stagnation point flow $(28)$,

$$
\bar{v}=\frac{x_{0}}{t_{\gamma}},
$$

to leading order in $2 \gamma t_{d 0}$. Magnetic feedback on the flow is unimportant if $\Delta v^{P} / \bar{v}<1$. Combining eqns. (51) and (53), we derive

$$
\frac{\Delta v^{P}}{\bar{v}}=\frac{B_{00}^{2}}{32 \pi \rho L^{2} \gamma^{2}}\left(2 \gamma t_{d 0}\right)^{1 / 2} \ln \left(2 \gamma t_{d 0}\right)
$$

Using eqns. (12) and (39), eqn. (54) can be rewritten as

$$
\frac{\Delta v^{P}}{\bar{v}}=\frac{\tau_{d}}{\tau_{A}}\left(\frac{\tau_{d}}{32 \tau_{n i}}\right)^{1 / 2} \ln \left(2 \frac{\tau_{A}^{2}}{\tau_{d} \tau_{n i}}\right) .
$$

Equation (55) implies an upper limit $\tau_{\max }^{P}$ on the eddy turnover time $\tau_{d}$ such that $\Delta v^{P} / \bar{v} \leq 1$;

$$
\frac{\tau_{\max }^{P}}{\tau_{A}}=\left(18 \frac{\tau_{n i}}{\tau_{A}}\right)^{1 / 3}\left[\ln \left(\frac{\tau_{A}}{\sqrt{2} \tau_{n i}}\right)\right]^{-2 / 3},
$$

where, to sufficient accuracy, we have replaced $\tau_{d}$ by $\left(32 \tau_{A}^{2} \tau_{n i}\right)^{1 / 3}$ in the logarithmic factor. On this timescale the magnetic field is still well frozen to the eddies; from eqns. (12) and (56),

$$
R_{A D}\left(\tau_{\max }^{P}\right)=\frac{v_{t}^{2}}{v_{A}^{2}}\left(18 \frac{\tau_{A}^{2}}{\tau_{n i}^{2}}\right)^{1 / 3}\left[\ln \left(\frac{\tau_{A}}{\sqrt{2} \tau_{n i}}\right)\right]^{-2 / 3} .
$$

\subsection{D Effects}

We assume the turbulent motions are in the $(x, y)$ plane, but depend weakly on $z$; i.e. the characteristic wavenumber $k$ along the field is related to the turbulent lengthscale $l$ by $k l \ll 1$. This quasi-two dimensionality is expected to be a feature of Alfvenic turbulence in strong magnetic fields (Strauss 1976, Goldreich \& Sridhar 1997).

Since the field is fairly well frozen in even on scales $l$, it is very well frozen on the scale $k^{-1}$, and the transverse field $\mathbf{B}_{\perp}$ is given to a good approximation by

$$
\mathbf{B}_{\perp}=B_{z}\left(\mathbf{x}_{\mathbf{0}}, t\right) \frac{\partial \mathbf{x}_{\perp}}{\partial z}
$$


where $\mathbf{x}_{\mathbf{0}}$ is the initial position of the fluid element at position $\mathbf{x}$ at time $t$.

Let us introduce a small parameter $\epsilon$ and assume that $\partial_{z}$ is $\mathcal{O}(\epsilon)$ relative to the perpendicular derivatives, and that $B_{\perp} / B_{z}$ is also $\mathcal{O}(\epsilon)$ (this is the so-called reduced MHD ordering; Strauss 1976). It can then be shown that the changes in the ambipolar drift terms are of order $\epsilon^{2}$. Therefore, we may assume that weak three dimensionality has little effect on ambipolar drift of the vertical field.

The bent field exerts a tension force which decelerates the fluid by an amount $\Delta v^{T}$. We compute $\Delta v^{T}$ for a $z$ dependent stagnation point flow model with

$$
\begin{gathered}
v_{x}=-\gamma x \cos k z, \\
v_{y}=\gamma y \cos k z,
\end{gathered}
$$

a generalization of eqns. (28). The Lagrangian positions are

$$
\begin{gathered}
x=x_{0} e^{-\gamma t \cos k z}, \\
y=y_{0} e^{\gamma t \cos k z} .
\end{gathered}
$$

According to eqns. (58) and (61), the $\hat{x}$ component of the field is

$$
B_{x}=B_{z} \gamma t k x \sin k z .
$$

The magnetic tension force $F_{m}$ in the $\hat{x}$ direction is

$$
F_{m}=\frac{B_{z}}{4 \pi} \frac{\partial B_{x}}{\partial z}=\frac{B_{z}^{2}}{4 \pi} \gamma t k^{2} x \cos k z
$$

We set $z=0$ and follow a procedure similar to the derivation of eqn. (55), integrating $F_{m}$ along the path of a fluid element from $t=0$ to $t=t_{\gamma}$. We approximate $B_{z}$ by $B_{00}$, which overestimates $F_{m}$. The result to leading order in $\left(2 \gamma t_{d 0}\right)^{-1}$ is

$$
\Delta v^{T}=\frac{1}{\rho} \int_{0}^{t_{\gamma}} d t F_{m}=\frac{k^{2} v_{A}^{2} x_{0}}{\gamma}
$$

Using eqn. (53), the relative deceleration is $\Delta v^{T} / \bar{v}$

$$
\frac{\Delta v^{T}}{\bar{v}}=\frac{k^{2} v_{A}^{2}}{2 \gamma^{2}} \ln \left(2 \gamma t_{d 0}\right) .
$$

Equation (66) shows that magnetic tension has little effect on the fluid as long as the Alfven frequency along the fieldline is less than the eddy turnover rate by the factor $\left[\ln \left(2 \gamma t_{d 0}\right)\right]^{1 / 2}$.

Equation (66) can be used to set an upper limit $\tau_{\max }^{T}$ on the eddy turnover time such that the magnetic field reaches the mixing scale without decelerating the fluid. Proceeding as in the derivation of eqn. (56) we find

$$
\frac{\tau_{\max }^{T}}{\tau_{A}}=\frac{\sqrt{2}}{k L}\left[\ln \left(\sqrt{2} k L \frac{\tau_{A}}{\tau_{n i}}\right)\right]^{-1 / 2} .
$$

Equation (67) shows that tension forces are less important in long, thin structures, in which $k L$ can be much less than one, than they are in flattened structures such as disks. The field is well frozen to the eddies as long as $\tau_{A} / \tau_{n i} \gg 1$. 


\section{Application to the Galactic Magnetic Field}

Little is know about interstellar turbulence beyond its gross energetics: the turbulent kinetic energy is at or above equipartition with the magnetic energy. Energy injection by a variety of mechanisms, combined with nonlinear processes, should lead to a turbulent spectrum over a wide range in scales.

In principle, any weakly ionized interstellar structure which survives for several eddy turnover times is a candidate for turbulent ambipolar diffusion. We showed in $\S 5$ that the efficiency of turbulent ambipolar drift can be limited by the back reaction of magnetic forces. In the strictly 2D case, the increase in ambipolar diffusion rate is associated with the local buildup of magnetic pressure forces, and in the 3D case, by magnetic tension forces as well. We expressed these constraints in terms of lower bounds on the strain rates, or inverse eddy turnover times, such that concentration of the field occurs before deceleration of the flow. These constraints appear in eqns. (56) and (67). Here, we express them numerically.

The critical parameters are the ratio $\tau_{n i} / \tau_{A}$ and the geometrical factor $k L$. Referring back to $\S 2.2$ for numerical values, we have

$$
\frac{\tau_{n i}}{\tau_{A}}=4.8 \times 10^{-5} \frac{B_{\mu}}{L_{p c} n_{i}\left(n_{n} A_{n}\right)^{1 / 2}},
$$

where $L$ is expressed in parsecs. For example, if $n_{n}=50 \mathrm{~cm}^{-3}, n_{i}=5 \times 10^{-3} \mathrm{~cm}^{-3}, A_{n}=1.4, L_{p c}=$ $1, B_{\mu}=3, \tau_{n i} / \tau_{A}=3.4 \times 10^{-3}$, and eqn. (56) requires $\tau_{d} / \tau_{A}<0.13$. If $v_{t} \sim v_{A}$, turbulence on the scale of a tenth of a parsec or less can mix the magnetic field down to the ambipolar diffusion scale. On the other hand, eqn. (67) requires $\tau_{d} / \tau_{A}<0.09(2 \pi / k L)$, which is a more severe constraint, especially in a highly flattened structure.

There is at least one type of H I structure in which flux freezing appears to be obeyed. Magnetic fields in H I shells are observed to be quite strong, with magnitudes consistent with shock compression (Heiles 1989). The same observations suggest that turbulence with Alfvenic or slightly

subAlfvenic velocities is present. If these shells were not expanding, they would appear to fulfill the conditions for fast ambipolar drift, and their strong fields would be counterexamples to the theory. However, expansion of the shells at speeds of order $10-20 \mathrm{~km} / \mathrm{s}$ adds new magnetic flux faster than it can diffuse upstream, while on the downstream side the ionization is too high for efficient ambipolar drift. Thus, the field in the shells remains large. Recent observations by Heiles (2001b) and Heiles \& Troland (2001) of cold, moderately dense H I regions which have weak magnetic fields, Alfvenic random velocities, and no observed association with shells appear to be better candidates for fast ambipolar drift. 


\section{Summary and Conclusions}

Observations show that while interstellar magnetic fieldstrength and gas density are to some extent correlated, the fieldstrength is often lower than expected. This suggests that processes beyond ideal MHD may play a role.

The flux to mass ratio is altered by ambipolar drift, but estimates of the ambipolar diffusivity $v_{A}^{2} \tau_{n i}$ predict that ambipolar drift is important only in very dense, strongly magnetized gas with substantial gradients on small scales. However, it is well established that turbulence can enhance the transport rates of quantities such as entropy and angular momentum. This motivated us to consider the effect of turbulence on the rate of ambipolar drift. Enhancement by roughly two orders of magnitude would explain the observations.

As a first attempt on the problem, we considered the geometrically restricted case of a straight magnetic field, with a transverse gradient, mixed by 2D, perpendicular turbulence. In this situation, ambipolar drift is described by a nonlinear diffusion term [eqn. (6)]. For simplicity, however, we approximated the diffusivity as linear.

We showed by a mixing length argument, and then an explicit calculation (§3) that advection of the field by a periodic flow reduces its peak value. However, unless the motions are long and thin, like streamers, the rate of relaxation is no faster than relaxation by ambipolar drift alone. The missing ingredient is stretching and shrinking of scales, which in chaotic flows happens at an exponential rate. In $\S 4$ we modelled these exponential changes of scale by representing the flow as a sequence of randomly oriented hyperbolic stagnation points. With this model, and a parabolic initial condition for the magnetic profile, the advection-diffusion equation (7) can be solved exactly. The model predicts an exponential increase of the mean diffusion rate with time (Figure 1), although with considerable variance from point to point (Figures $2 \& 3$ ). The stagnation point model predicts that the field diffuses on a timescale comparable to the eddy turnover time, with only logarithmic dependence on the ambipolar diffusivity itself and on the original gradient lengthscale.

In $\S 5$, we estimated the back reaction of magnetic pressure and tension forces on the stagnation point flow, including weak three dimensionality. The relative deceleration of the fluid over one mixing time is given for pressure forces by eqn. (54) and for tension forces by eqn. (66). Comparing the deceleration time of a fluid element to the mixing time, we derived upper limits on the eddy turnover time such that deceleration is order unity or less within a mixing time [eqns. (56) and (67)]. As we showed in $\S 6$, these criteria can be satisfied in the interstellar medium without extreme assumptions about the size and velocity of the turbulent eddies, although they cannot be wholly ignored. These estimates of feedback are conservative in the sense that a fully self consistent model of MHD turbulence can still produce fast mixing, as shown by Maron \& Goldreich (2001) for spreading of a passive scalar. This conclusion may be dependent on geometry, however, as concluded by Kim (1997) based on a study of turbulent decay of a coplanar magnetic field caused by $2 \mathrm{D}$ incompressible motions in weakly ionized fluid. 
The outcome of these calculations is that turbulence is likely to have a major effect on the magnetic flux to mass ratio in the weakly ionized portions of the interstellar medium, making the magnetic field more uniform. The model presented here applies to regions with simple magnetic topology, filamentary structure, and no global cross-field flows. It follows that the strength of the field is not necessarily a good indicator of the dynamical processes which determine the gas density.

Although the results presented here are consistent with the conventional wisdom that turbulent mixing takes place in an eddy turnover time, it is important to recognize that they are not obtained from a full model of turbulence. We plan to extend this work to more realistic models which include compressibility, vorticity, and a self consistent treatment of magnetic forces as well as more general magnetic geometry. If the stagnation point model holds up in comparison with more complete models, it could be useful in other mixing problems.

Magnetic reconnection can also change the magnetic flux to mass ratio. Lazarian \& Vishniac (1999) have argued that if the spectrum of interstellar turbulence extends to the resistive scale then reconnection takes place at the Alfven speed. We have concentrated here on ambipolar drift because it does not require turbulent structure on such small scales; if flux is quickly redistributed in the fully ionized portions of the ISM then an alternative process is certainly required.

This work was initiated during the program on Astrophysical Turbulence at the ITP in Santa Barbara in 2000. I am happy to acknowledge useful discussions with Nic Brummell, Dick Crutcher, George Field, Carl Heiles, Fabian Heitsch, David Hughes, Eun-jin Kim, Steve Tobias, Tom Troland, and especially the referee, Pat Diamond. Material support was provided by NSF Grants AST 9800616 and AST 0098701 to the University of Colorado and PHY 9407194 to UC Santa Barbara.

\section{REFERENCES}

Bourke, T.L., Myers, P.C., Robinson, G., \& Hyland, A.R. 2001, ApJ, in press, and astro-ph 0102469

Brandenburg, A., \& Zweibel, E.G. 1994, ApJ, 427, L91

Brandenburg, A., \& Zweibel, E.G. 1995, ApJ, 448, 734

Brogan, C. \& Troland, T. 2001, ApJ, 550, 799

Cattaneo, F. 1994, ApJ, 434, 200

Cattaneo, F., Hughes, D.W., \& Kim, E. 1996, Phys. Rev. Lett., 76, 2057

Cattaneo, F., Kim, E., Proctor, M.R.E., \& Tao, L. 1995, Phys. Rev. Lett., 75, 1522

Cattaneo, F., \& Vainshtein, S. 1991, ApJ, 376, L21

Childress, S. \& Gilbert, A.D. 1995, Stretch, Twist, Fold: The Fast Dynamo, Springer 
Crutcher, R.M. 1999, ApJ, 520, 706

Draine, B.T., Roberge, W.R., \& Dalgarno, A. 1983, ApJ, 264, 485

Galloway, D.J. \& Proctor, M.R.E. 1992, Nature, 356, 691

Goldreich, P.M., \& Sridhar, S. 1997, ApJ, 485, 680

Gruzinov, A., \& Diamond, P.H. 1994, Phys. Rev. Lett., 72, 1651

Heiles, C. 1989, ApJ, 336, 808

Heiles, C. 2001a, to be published in Proceedings of the 4th Tetons Summer Conference, and astro-ph 0010047

Heiles, C. 2001b, ApJ, 551, L105

Heiles, C., \& Troland, T. 2001, in preparation

Kim, E. 1997, ApJ, 477, 183

Lazarian, A., \& Vishniac, E.T. 1999, ApJ, 517, 700

Mestel, L. 1985, in Protostars \& Planets II, eds. D.C. Black \& M.S. Matthews, Univ. Arizona Press, p. 320

Mestel, L., \& Spitzer, L.S. 1956, MNRAS, 116, 505

Moffatt, H.K. 1978, Magnetic Field Generation in Electrically Conducting Fluids, Cambridge Univ. Press

Mouschovias, T. 1976, ApJ, 207, 141

Ottino, J.M. 1989, The Kinematics of Mixing, Cambridge Univ. Press

Padoan, P. \& Nordlund, A. 1999, ApJ, 526, 279

Ponty, Y.,Pouquet, A., Rom-Kedar, V., \& Sulem, P.-L. 1993, in Solar \& Planetary Dynamos, eds. M. Proctor, P. Matthews, \& A. Rucklidge, Cambridge Univ. Press, p. 241

Press, W.H. \& Rybicki, G. 1981, ApJ, 248, 751

Shraiman, B.I. \& Siggia, E.D. 2000, Nature, 405, 639

Shu, F.H. 1983, ApJ, 273, 202

Sridhar, S., \& Goldreich, P.M. 1994, ApJ, 432, 612

Strauss, H.R. 1976, Phys. Fluids, 19, 134 
Tomisaka, K., Ikeuchi, C., \& Nakamura, T. 1988, ApJ, 335, 239

Troland, T.H. \& Heiles, C. 1986, ApJ, 301, 339

Zel'dovich, Y.B.\& Raizer, Y.P. 1966, Physics of Shock Waves \& High Temperature Hydrodynamic Phenomena, Academic Press

Zel'dovich, Y.B., Ruzmaikin, A.A., Molchanov, S.A., \& Sokoloff, D.D. 1984, J. Fluid Mech., 144, 1

Zweibel, E.G. 1988, ApJ, 329, 384

Zweibel, E.G. 1998, Phys. Plasmas, 5, 247

Zweibel, E.G. \& Brandenburg, A. 1997, ApJ, 478, 563 